\title{
The underhood aerothermal management of leakage zones: temperature measurements
}

\author{
M. Khaled ${ }^{1,2}$, M. Ramadan ${ }^{1}$, A. Elmarakbi ${ }^{3}$, \\ F. Harambat ${ }^{4} \&$ H. Peerhossaini ${ }^{2}$ \\ ${ }^{1}$ School of Engineering, Lebanese International University, Lebanon \\ ${ }^{2}$ Energy Research Institute (PIERI), France \\ ${ }^{3}$ University of Sunderland, $U K$ \\ ${ }^{4}$ PSA Peugeot Citroën, France
}

\begin{abstract}
The leakage areas of the engine compartment are located essentially at the junctions between the hood and the front end, the sides, the lights, and the windshield. The present study concerns the impact of these leakage zones on the engine compartment temperatures. The study synthesizes thermal measurements carried out on a real vehicle in the wind tunnel S4 of Saint-Cyr l'Ecole France. The engine compartment is instrumented with 80 surface and air thermocouples. Measurements are carried out for three different thermal functioning points with the front wheels of the vehicle positioned on a dynamometer and driven by the engine of the car. Five configurations of leakages closing are tested.
\end{abstract}

Keywords: underhood, aerothermal management, leakages, temperature, measurement.

\section{Introduction}

To increase the engine efficiency of vehicle, previous works has suggested that one must mainly reduce the energy lost in the exhaust system or use some of it [1], increase and/or control cooling-system efficiency [2-3], reduce the energy consumption of the HVAC system, or improve the underhood (engine) design [4-5]. However, little work has been done on aerothermal effects that could improve energy-balance management in a well-controlled vehicle [6-7]. In this 
context, the present study highlights the relation between an important kind of aerothermal effect, the leakage zones, and the thermal management of the underhood.

The leakage areas of the engine compartment are located essentially at the junctions between the hood and the front end, the sides, the lights, and the windshield. Often these leakages are not well controlled experimentally or modeled numerically. Aerodynamic studies have shown that the influence of the leakage on the cooling drag can be considered negligible. Thus, not taken into account leakages in aerodynamic calculations do not impact the results. However, in thermal management, two questions arise: under normal driving conditions, does an uncontrolled airflow due to the leakage zones impact the thermal situation? Under conditions at the vehicle stopping, do the leakage zones have a positive effect on the underhood cooling, especially in the thermal soak phase?

To answer these questions, it proved important to test the effect of leakage areas on the thermal situation under the hood in order to assess their impact on the underhood aerothermal management. At this point, two studies revealed necessary for judgment: the first being to check if leakage zones impact the underhood temperatures (component, air zones, and engine parameters) in both constant-speed driving and thermal soak phases, and the second of comparing the temperature differences due to leakages to the accuracy of numerical calculations.

In this paper, an experimental study on a vehicle, where temperatures in the under-hood for different configurations of closing leakage zones are compared, is presented. In section 2, the test procedure is exposed: in particular instrumentation, control parameters and details of the configurations. Then, the results and the methods used (sections 3 and 4) for the analysis of the effects of leakages on underhood temperatures are presented. Finally, section 5 draws the main conclusions of the work.

\section{Tests description}

In this section, the instrumentation (section 2.1), the test configurations (section 2.2), the different phases of a test (section 2.3), and the test protocol with the different phases (section 2.4) are presented.

\subsection{Instrumentation}

The underhood of the car used for the tests is instrumented with surface and air thermocouples of type $\mathrm{T}$ and type $\mathrm{K}$. These thermocouples allow the measurement of temperatures of about 80 locations, corresponding to temperatures of components, air zones and engine parameters. Among the components studied, the most important are the exhaust manifold, intake manifold, cold box (area comprising the computer and battery), apron, water outlet plenum and alternator. For air areas, the most important are those of the cold box, crawl, apron, engine downstream, fan downstream and charge air 
cooler CAC downstream. The engine parameters are essentially temperatures at the inlet and outlet of the radiator, the $\mathrm{CAC}$, the turbo, the catalyst and the engine inlet temperature.

\subsection{Test configurations}

Thermal tests are performed at wind tunnel S4 of Saint-Cyr l'Ecole-France. Three wind speeds in the tunnel are tested. The front wheels are placed on a dynamometer. The car engine is running. A driver in the vehicle accelerates and the front wheels drive the rollers. The dynamometer has a "brake" system, allowing the adjustment and control of the power to the wheels and the speed of rotation. The tests are carried out for three points of different thermal operation, described in Table 1 and called thermal functioning points. In Table 1 , $\mathrm{V}_{\text {Wheel }}$ and $\mathrm{V}_{\text {wind }}$ are respectively the wheel and wind speeds.

Table 1: Parameters defining the three thermal functioning points.

\begin{tabular}{ccccc}
\hline & $\begin{array}{c}\mathbf{V}_{\text {Wheel }} \\
\mathbf{K m}^{\mathbf{- 1}}\end{array}$ & $\begin{array}{c}\mathbf{V}_{\text {wind }} \\
\mathbf{K m}_{\mathbf{h}^{\mathbf{1}}}\end{array}$ & $\begin{array}{c}\text { Gear ratio } \\
-\end{array}$ & $\begin{array}{c}\text { Engine } \\
\mathbf{r p m}\end{array}$ \\
\hline TFP-1 & 90 & 90 & 5 & 2600 \\
TFP-2 & 110 & 55 & 4 & 3800 \\
TFP-3 & 130 & 130 & 5 & 3780 \\
\hline
\end{tabular}

To test the effect of leakage, five configurations of leakage zones closing are studied: all leakage zones open, only the junction hood-front end closed, the junction hood-sides closed in addition, the junction hood-lights closed in addition, and all zones closed.

A software connected to the computer of the car is used to control the fan. The latter has two speeds. The software keeps the fan at high speed during the constant-speed driving phase. At the vehicle stopping (early thermal soak), the computer returns to the stand-alone mode and the fan turns at its low speed for few minutes before shutting down completely.

\subsection{Different phases of a test}

For each test (thermal functioning point and leakages configuration fixed), the temperature recording covers three successive phases, each simulating a real situation to which a car can be confronted: constant-speed driving, slow-down and thermal soak.

1. The constant-speed driving phase represents the actual car driving at an engine speed, a gear ratio and at given forward and wind speeds. This constant-speed driving phase can be either TFP-1 or TFP-2 or TFP-3.

2. The slowdown phase is simulated in the wind tunnel by passing to the neutral after the rolling phase and stopping the wind in the wind tunnel.

3. The thermal soak phase follows the slow-down phase and simulates the vehicle stopping after a high thermal load. The thermal inertia of the 
underhood components maintains high temperatures despite the extinction of the engine. In this case, the circulation of air for cooling the underhood is only due to the rotation of the fan and/or natural convection. At the beginning of the thermal soak, the fan runs for a short time (1-5 minutes) before stopping.

\subsection{Test protocol with the different phases}

For a given configuration of leakages closure, first the thermal functioning point is stabilized (TFP-1, TFP-2 or TFP-3). The engine speed then reaches the preset value and the acquisition is started to save the 3 phases of constant-speed driving, slow-down and thermal soak. The constant-speed driving phase is maintained until the temperature stabilization. The slowdown occurs then when the driver cowards the accelerator: the wind in the wind tunnel is cut and the car engine is turned off when the air velocity in the wind tunnel reaches zero. The moment of engine extinction corresponds to the beginning of the thermal soak phase. During the recording, the beginning of each of the three phases is detected.

\section{Qualitative analysis: results}

A first analysis of the results is made with a "qualitative" method: it consists on emerging the typical trends of temperature variations as a function of the leakage zones. This method consists in a first time on separating the records of the various phases and focusing on the two phases of interest: the constant-speed driving and thermal soak phases.

To study the effects of leakage zones on a given location, the temporal variation of the temperature for five configurations of leakage closing are traced by readjusting the curves with respect to the time of stabilization of the engine speed in constant-speed driving phase and to the moment of stopping the engine in the thermal soak phase.

By applying this method to the various components, air zones, and engine parameters, the following typical trends are noticed:

- The closure of the junctions hood-front end, hood-wings and hoodwindshield increases significantly the temperature of many components, air zones and engine parameters, in both constant-speed driving and thermal soak phases.

- These effects are much more sensitive to the two junctions' hood-front end and hood-windshield than for the junction hood-sides.

- Surprisingly, the trend is reversed for the closure of the hood-lights junction: its closure reduces temperatures.

To illustrate these results and provide orders of magnitude, Figure 1 shows typical temperature trends on the water outlet plenum and the air near the apron area. 
Configuration 0 corresponds to the configuration where all leakage zones are open. Configuration 1 corresponds to the one where the junction hood-front end is blocked. Configuration 2 corresponds to the configuration 1 with in addition the junction hood-sides closed. Configuration 3 corresponds to the configuration 2 with in addition the junction hood-lights closed. Finally, Configuration 4 corresponds to the configuration 3 with in addition the hood-windshield closed ("all leakages closed" configuration).

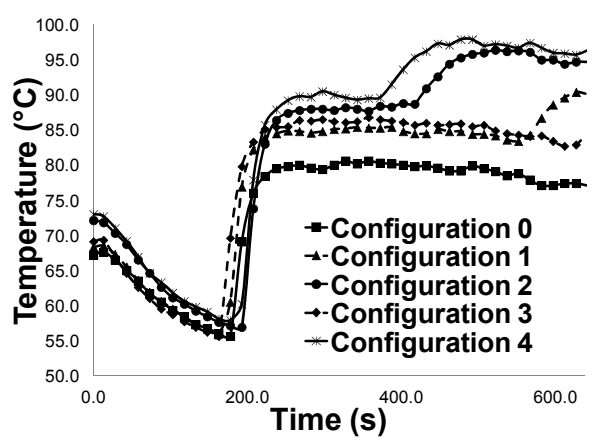

(a)

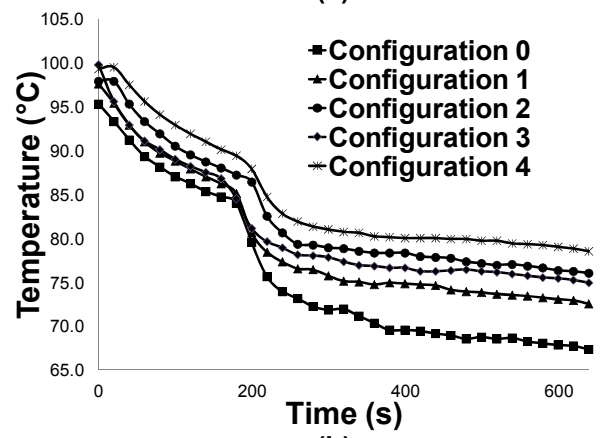

(b)

Figure 1: Leakage effects on the temperature during the thermal-soak phase: (a) water outlet plenum, (b) the air zone near the apron.

For the water outlet plenum (WOP), one clearly notes the leakage effects on the temperature at the end of the thermal soak and for very close initial temperatures (Figure 1(a)). At the end of the thermal soak phase, with respect to a temperature of $76.7^{\circ} \mathrm{C}$ for all the leakage zones open, a local temperature rise of $13.5^{\circ} \mathrm{C}$ was measured by closing the junction hood-front end. The temperature increases by $4.5^{\circ} \mathrm{C}$ when the junction hood-sides is closed and $10.4^{\circ} \mathrm{C}$ when the junction hood-windshield is blocked. These trends are also observed on other components. Conversely, the closing of the junction hood-lights causes a cooling of the WOP of $-9.3^{\circ} \mathrm{C}$. This cooling is also found for all components and air zones. 
This result is explained by the fact that the junction hood-lights is an area of depression and therefore corresponds to an underhood air outlet (this non-trivial behavior has already been observed in numerical calculations on other vehicles). By closing this junction, low flows of fresh air entering the underhood and to be exiting through the hood-lights zone are denied. Since the airflow cannot get out by this junction, it tends to move closer to the components and contributes to better cooling thereof.

These thermal behaviors are found on other components and air areas, such as near the apron area (Figure 1(b)). For a temperature of $67.2^{\circ} \mathrm{C}$ at the end of the thermal soak, differences of $5.2^{\circ} \mathrm{C}, 3.6^{\circ} \mathrm{C}, 1.2^{\circ} \mathrm{C}$ and $3.6^{\circ} \mathrm{C}$ are measured respectively for configurations $1,2,3$, and 4 .

For the constant-speed driving phase, the same effects are shown for the different areas of leakage, but with much smaller differences. Moreover, these differences are sometimes in the same order of magnitude as the differences between the initial temperatures and their interpretation becomes hazardous (Figure 2).

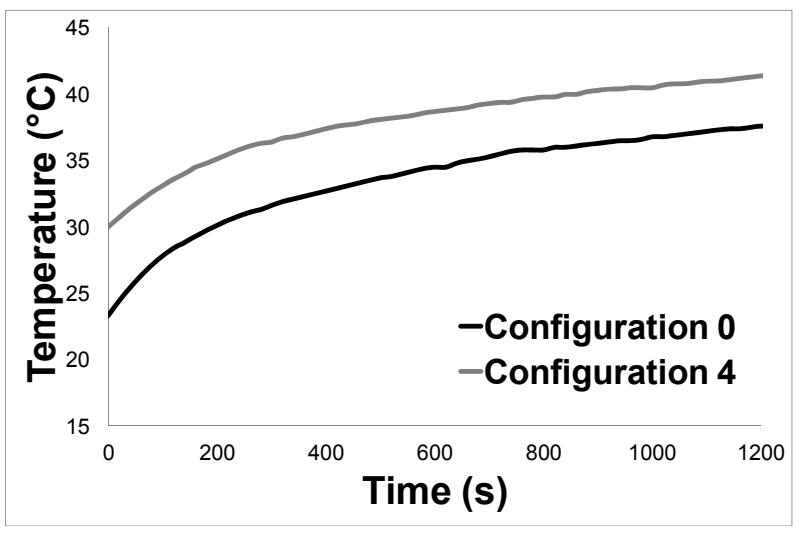

Figure 2: Leakage effects on the temperature of the triangle (relating the transmission to the wheels) in constant-speed driving phase for TFP-3.

To free ourselves from defects due to different initial conditions of each phase, we propose a so-called "quantitative analysis" method.

\section{Quantitative analysis: results}

Having the same initial conditions of temperatures at the beginning of each phase is practically impossible. In addition, at the beginning of the thermal soak, the fan starts rotating at its secondary speed for a short period (before shutting down completely) and this time depends on the configuration of leakages 
blocking and operating point. Therefore, in order to quantitatively compare the different configurations, we use a method of analysis that includes:

1. Separate the different phases of the test (constant-speed driving, slowdown and thermal soak);

2. Separate the strictly monotone parts in the thermal soak (this eliminates the effect of operating time of the fan at its low speed);

3. Match the initial conditions in each part obtained, and calculate the relative deviation of final temperatures.

The method is applied to the set of components and air zones tested in order to quantify the effect of air leakage areas. Figure 3 shows the maximum relative differences on all the separate parts for each component, in thermal soak and constant-speed driving phases.
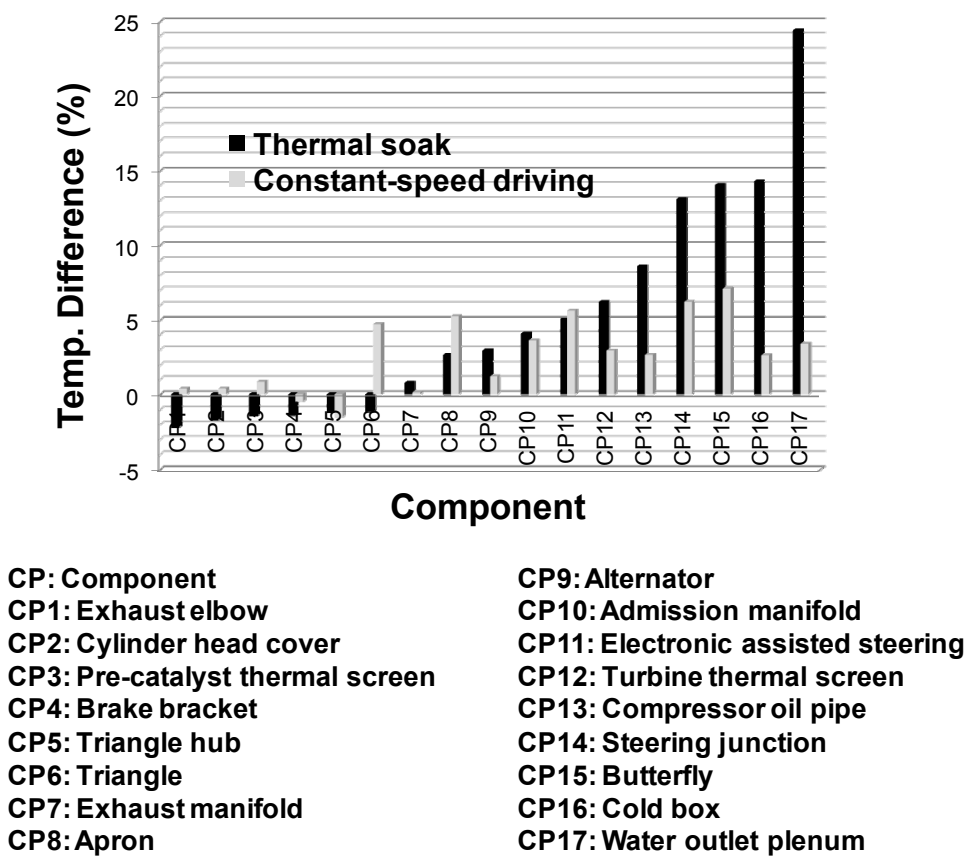

Figure 3: Temperature relative differences due to leakage closure for different components in TFP-2 for both thermal soak and constantspeed driving phases.

The relative temperature difference due to closing all the leakage zones exceeds $4 \%$ for most of the components in constant-speed driving phase (for example $6 \%$ at the steering junction and $7 \%$ at the throttle). In thermal soak, 
these differences are higher. They exceed $6 \%$ for the same components (for example they are $14 \%$ at the cold box and $24 \%$ at the water outlet plenum).

Figure 4 shows the relative temperature differences due to leakage closing at the different air zones.

In thermal soak, higher differences can be seen at air zones than at components. These differences exceed $10 \%$ for almost all studied air zones. They reach the $25 \%$ level at the cold box zone and $30 \%$ at the wings. When driving, the differences are much lower (for example $6.2 \%$ at the brake disc and $7.6 \%$ at the wings).

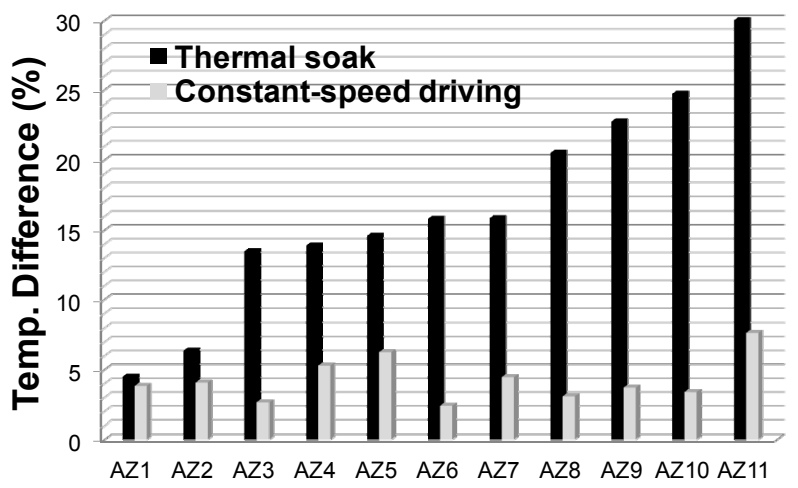

Air Zone

AZ: Air zone

AZ1: Engine downstream

AZ2: Radiator downstream

AZ3: Crawl

AZ4: Wheel arch

AZ5: Brake disk
AZ6: Apron
AZ7: Steering junction
AZ8: Charge air cooler downstream
AZ9: Fan downstream
AZ10: Cold box
AZ11: Underhood sides

Figure 4: Temperature relative differences due to leakage closure for different air zones in TFP-2 for thermal soak and constant-speed driving phases.

\section{Conclusions}

Leakage zones have a significant effect on the underhood thermal situation, in both constant-speed driving and thermal soak phases. These effects are most notable in thermal soak and clearly significant (deviations from 6 to 30\%, depending on the location). The leakage zones at the hood-front end, hood-sides and hood-windshield reduce temperatures in the engine compartment: these decreases are more sensitive to the two junctions hood-front end and hoodwindshield than to the hood-sides junction. Inversely and in a non-trivial way, the junction hood-lights increases the temperatures in the engine compartment. 


\section{References}

[1] Manzela, A.A, Hanriot, S.M, Cabezas-Gomez, L., \& Sodre, J.R., Using engine exhaust gas as energy source for an absorption refrigeration system, Applied Energy, 87, pp. 1141-1148, 2010.

[2] Khaled, M., Harambat, F., El Hage, H., \& Peerhossaini, H., Spatial optimization of an underhood cooling module - towards an innovative control approach, Applied Energy, 88, pp. 3841-3849, 2011.

[3] Khaled, M., Mangi, F., El Hage, H., Harambat, F., \& Peerhossaini, H., Fan air flow analysis and heat transfer enhancement of vehicle underhood cooling system - towards a new control approach for fuel consumption reduction, Applied Energy, 91, pp. 439-450, 2012.

[4] Jung, D., Kwak, K.H., \& Assanis, D.N., Integration of a single cylinder engine model and a boost system model for efficient numerical mapping of engine performance and fuel consumption, International Journal of Automotive Technology, 13, pp. 1-7, 2012.

[5] Khaled, M., Harambat, F., \& Peerhossaini, H., Towards the control of car underhood thermal conditions, Applied Thermal Engineering, 31, pp. 902-910, 2011.

[6] Khaled, M., Al Shaer, A., Hachem, F., Harambat, F., \& Peerhossaini, H., Effects of ground vehicle inclination on underhood compartment cooling. International Journal of Automotive Technology, 13, pp. 895-904, 2012.

[7] Khaled, M., Harambat, F., \& Peerhossaini, H., A quantitative method for the assessment of car inclination effects on thermal management of the underhood compartment, Journal of Thermal Science and Engineering Applications, ASME, 1:014501, pp. 1-5, 2009. 